\title{
Variant analysis of prostate cancer in Japanese patients and a new attempt to predict related biological pathways
}

\author{
RIKA KASAJIMA $^{1,2}$, RUI YAMAGUCHI ${ }^{3,8}$, EIGO SHIMIZU ${ }^{3}$, YOSHINORI TAMADA ${ }^{3,9}$, \\ ATSUSHI NIIDA $^{4}$, GEORGE TREMMEL ${ }^{3}$, TAKESHI KISHIDA ${ }^{5}$, ICHIRO AOKI ${ }^{6}$, SEIYA IMOTO ${ }^{2}$, \\ SATORU MIYANO ${ }^{3,4}$, HIROJI UEMURA ${ }^{7}$ and YOHEI MIYAGI ${ }^{1}$
}

\begin{abstract}
${ }^{1}$ Molecular Pathology and Genetics Division, Kanagawa Cancer Center Research Institute, Yokohama, Kanagawa 241-8515;
${ }^{2}$ Division of Health Medical Data Science, Health Intelligence Center; ${ }^{3}$ Laboratory of DNA Information Analysis, Human Genome Center; ${ }^{4}$ Division of Health Medical Computational Science, Health Intelligence Center, Institute of Medical Science, University of Tokyo, Tokyo 108-8639; 5 Department of Urology, Kanagawa Cancer Center Hospital, Yokohama, Kanagawa 241-8515; ${ }^{6}$ Niwa Hospital Pathology Section, Odawara, Kanagawa 205-0042; ${ }^{7}$ Department of Urology and Renal Transplantation, Yokohama City University Medical Center, Yokohama, Kanagawa 236-0027, Japan
\end{abstract}

Received May 8, 2019; Accepted November 12, 2019

DOI: $10.3892 /$ or.2020.7481

\begin{abstract}
There are regional and/or ethnic differences in tumorigenic pathways among several types of cancer, including prostate cancer (PCa). However, information on genome-wide gene alterations and the transcriptome is currently only available for PCa patients from Western countries. In order to profile the genetic alterations in Japanese patients with PCa, new panels were created to examine nucleotide sequence variations in 71 selected PCa-related genes (KCC71) and to detect all fusion RNA transcripts known in $\mathrm{PCa}$ (PCaFusion). An analysis of 21 Japanese PCa cases identified 33 different somatic variants in 24 genes in the KCC71 panel, including 2 in SPOP (F102V and F133L), 2 in BRCA2 (I1859fs and $\mathrm{R} 2318$ ter, resulting in premature termination of the polypeptide), and 1 each in BRAF (K601E), CDHI (E880K) and $R B 1$ (R621S), as pathogenic alterations. Unexpectedly, the $T M P R S S 2-E R G$ fusion transcript was detected in only 1 case, although the SLC45A3-ELK4 and USP9Y-TTTY15 fusion transcripts, known as transcription-mediated chimeric RNAs, were detected in all examined cases. A new pathway analysis with The Cancer Network Galaxy (TCNG), a cancer gene regulatory network database, was also applied in an attempt to
\end{abstract}

Correspondence to: Dr Yohei Miyagi, Molecular Pathology and Genetics Division, Kanagawa Cancer Center Research Institute, 2-3-2 Nakao, Asahiku, Yokohama, Kanagawa 241-8515, Japan

E-mail: miyagi@gancen.asahi.yokohama.jp

Present addresses: ${ }^{8}$ Division of Cancer System Biology, Aichi Cancer Center Research Institute, Nagoya, Aichi 464-8681, Japan; ${ }^{9}$ Department of Medical Intelligent Systems, Graduate School of Medicine, Kyoto University, Kyoto 606-8507, Japan

Key words: prostate, cancer, Japanese, mutation, fusion, next generation sequencing, gene regulatory network, gene set enrichment analysis predict molecular pathways implicated in PCa in the Japanese population. Based on the 24 genes having somatic variants identified by the panel analysis as initial seed genes, a putative core network was finally established, including 5 identified genes, namely TNK2, SOX9, CDH1, FOXA1 and TP53, with high commonality from TCNG datasets. These genes are expected to be involved in tumor development, as revealed by the results of an enrichment analysis with Gene Ontology terms. This analysis must be further extended to include more cases in order to verify this method and also to elucidate the characteristics of PCa in Japanese patients.

\section{Introduction}

Several integrated analyses of whole-genome and whole-exome sequencing data and transcriptomics have been reported for cohorts of prostate cancer (PCa) in Western countries. In these cohorts, the incidence of androgen-inducible fusion oncogenes generated by chromosomal alterations involving erythroblastosis virus E26 transformation-specific related gene $(E R G)$ was reported to be $>50 \%$ (1-4). In addition to $E R G$-associated fusion events, variants of SPOP and MEDI2 and deletions of chromosome 5q21/6q21 have been reported as common genomic alterations $(5,6)$. Recently, BRCA1, BRCA2 $(5,7)$ and $H O X B 13(5,8)$ were identified as new therapeutic targets or tumor markers, on which new molecular pathway analyses are currently being conducted.

We previously reported that $\mathrm{PCa}$ harboring the $T M P R S S 2-E R G$ fusion gene was less frequent in Japan compared with Western countries (9). Another group supported this finding in an independent Japanese cohort together with a Chinese cohort, suggesting that this low frequency is characteristic of PCa in Asians (10). Although the exact frequency of $S P O P$ variations has not yet been determined in Japanese patients, TMPRSS2-ERG and SPOP variations occur in a mutually exclusive manner in Western countries $(6,11)$, and it has been reported that there may be a clinical benefit 
in classifying patients into TMPRSS2-ERG-positive and $S P O P$-mutated groups (9). This background suggests the potential benefits of also performing thorough investigations of the genomic alterations in Japanese patients, as well as in patients from Western countries.

It has been indicated that the profiling of genetic alterations alone is insufficient to obtain a comprehensive understanding of the tumorigenesis pathway; trans-omics studies are required for this purpose. However, such studies are resource-intensive due to the need for genomic, transcriptomic, proteomic, epigenetic, or more omics analyses on the same tumor specimen, followed by integration of the results and identification of the biological pathways. In the present study, the gene network model data of The Cancer Network Galaxy (TCNG; Human Genome Center, University of Tokyo; http://tcng.hgc. jp/index.html) was used to deduce the characteristics of PCa in the Japanese population, using the limited gene variation data that were obtained in the present study. TCNG is a database of gene networks estimated from high-throughput biological data using a Bayesian network $(12,13)$. Some genetic variants disrupt the balance of the regulatory relationships between genes, which may result in cancer; as such, if this approach is extended to include a higher number of cases, the above analyses may enable a comprehensive overview of PCa in Japanese patients.

\section{Materials and methods}

PCa patients and tumor specimens. A total of 21 PCa patients who underwent radical prostatectomy between 2011 and 2014 at the Department of Urology, Yokohama City University Graduate School of Medicine, were included in the present study. Several parts of each resected prostate that had been indicated to contain cancer tissues by preoperative examinations were embedded in OCT compound (Sakura Finetek Japan) and immediately stored at $-80^{\circ} \mathrm{C}$. The patient clinical information is summarized in Table I. All the patients were Japanese, with a mean age of 67 years (range, 51-76 years), and serum prostate-specific antigen (PSA) values ranging from 4.4 to $31.0 \mathrm{ng} / \mathrm{ml}$ (mean, $10.4 \pm 7.36 \mathrm{ng} / \mathrm{ml}$ ). All tumors were diagnosed as non-metastatic adenocarcinomas and assigned a Gleason score of 6-9 at the Department of Pathology. When multiple Gleason scores had been assigned to one patient, the highest score was used, as shown in Table I.

Design of the original panels for detection of genetic alterations. In order to profile the genetic alterations in Japanese patients with PCa in an efficient as well as highly sensitive manner, two original panels were prepared for the targeted sequencing of genes that were reported to be altered in previous whole-genome or whole-exome sequencing studies in Western countries. The KCC71 panel was for DNA samples designed to detect single-nucleotide variations (SNVs) and small insertions and deletions (indels) in $71 \mathrm{PCa}-$ related genes and driver genes reported by whole-exome and whole-genome sequencing $(4-11,14,15)$ (Table SI). The PCaFusion panel was for RNA samples designed to detect transcripts derived from 38 previously reported fusion transcripts, together with 8 control transcripts (Table SI). In addition, the PCaFusion panel was designed to detect fusion transcripts with different exonic junctions from the same fusion partners (1-5,14-18). The multiplex-PCR primer sets for the KCC71 and PCaFusion panels are provided in Tables SIIA and SIIB.

Sample preparation and target sequencing with the original panels. To obtain DNA and RNA samples, a thin-sliced section was prepared from each stored frozen specimen, embedded in OCT compound and stained with hematoxylin and eosin (HE). Based on information on the area of tumor tissues in the HE-stained section, PCa tissues were obtained directly from the remaining OCT-embedded specimen, from which DNA and RNA were extracted using ZR-Duet DNA/RNA miniprep (Zymo Research), following the manufacturer's protocol. DNA and RNA were quantified with Qubit 2 (Thermo Fisher Scientific, Inc.). To assess the DNA and RNA quality, ratios of optical densities, A260/A280 and A260/A230, were further evaluated by NanoPhotometer (Implen). A total of $10 \mathrm{ng}$ of genomic DNA or total RNA was used to create panel libraries for each specimen. Library amplification was performed using Ion Torrent AmpliSeq ${ }^{\mathrm{TM}}$ technology, along with sequencing with the Ion PGM next-generation sequencer (Thermo Fisher Scientific, Inc.).

Variant call and validation. Torrent Suite v4.0.2 and Ion Reporter version 4.4 (Thermo Fisher Scientific, Inc.) softwares were used to process and analyze the sequenced data from the Ion PGM. Quality control reports were obtained from the Torrent Suite. To identify somatic variants, the SNVs and indels with a coverage rate of $\geq 20$ and with coding amino acid sequence substitutions when compared with the UCSC hg19 reference genome sequence were first selected. Next, single-nucleotide polymorphisms (SNPs) were excluded by using the sequences as queries against the data in the databases COSMIC (http://cancer.sanger.ac.uk/cosmic), dbSNPs (NCBI, NIH; https://www.ncbi.nlm.nih.gov/projects/SNP/), the 1000 Genomes Project (http://www.1000genomes.org), and other publicly accessible databases. For SNVs for which it remained unclear whether they were SNPs or somatic variants after database analysis, Sanger sequencing on DNA from the non-neoplastic counterpart of each specimen was performed to obtain definitive results. Finally, sequence alterations with an allele frequency of $>5 \%$ were defined as somatic variants in the present study.

Fusion transcript detection. Torrent Suite v4.0.2 and Ion Reporter version 4.4 were used to process and analyze the sequenced data from the PCaFusion panel. Quality Check reports were obtained from the Torrent Suite server. The unclear fusion transcripts identified by the PCaFusion panel were further verified by reverse-transcription (RT)-PCR followed by Sanger sequencing of the products.

\section{Results}

Sequencing statistics. A summary of the sequencing statistics is presented as a representative case of KCC71 panel analysis in Fig. S1A. The means of the obtained reads and coverage were $3,902,663$ and 1,850, respectively. The data on average alignment ratios revealed that $97.6 \%$ of the total reads were aligned properly to the hg19 human genome reference sequence. A 
Table I. Clinical information for 21 prostate cancer patients.

\begin{tabular}{|c|c|c|c|c|c|}
\hline Case & Age (years) & $\mathrm{pTNM}^{\mathrm{a}}$ & Gleason Score ${ }^{b}$ & Histology & PSA (ng/ml) \\
\hline 1 & 65 & pT2cNOM0 & $3+3=6$ & Adenocarcinoma & 7.2 \\
\hline 2 & 69 & pT3aNOM0 & $4+5=9$ & Adenocarcinoma & 11.0 \\
\hline 3 & 62 & pT2cNOM0 & $3+4=7$ & Adenocarcinoma & 5.6 \\
\hline 4 & 76 & pT2cN0M0 & $3+4=7$ & Adenocarcinoma & 8.2 \\
\hline 5 & 63 & pT3b, N0 & $4+3=7$ & Adenocarcinoma & 14.0 \\
\hline 6 & 56 & pT2cNOM0 & $3+4=7$ & Adenocarcinoma & 4.4 \\
\hline 7 & 61 & pT2cN0M0 & $4+3=7$ & Adenocarcinoma & 5.3 \\
\hline 8 & 71 & pT3aN0M0 & $3+4=7$ & Adenocarcinoma & 15.2 \\
\hline 9 & 76 & pT3aN0M0 & $3+4=7$ & Adenocarcinoma & 15.6 \\
\hline 10 & 59 & pT2cN0M0 & $4+4=8$ & Adenocarcinoma & 11.4 \\
\hline 11 & 75 & pT2cN0M0 & $4+5=9$ & Adenocarcinoma & 5.6 \\
\hline 12 & 65 & pT3aN0M0 & $3+4=7$ & Adenocarcinoma & 5.4 \\
\hline 13 & 71 & pT3aN0M0 & $4+5=9$ & Adenocarcinoma & 31.0 \\
\hline 14 & 71 & pT2cN0M0 & $4+4=8$ & Ductal adenocarcinoma & 7.6 \\
\hline 15 & 71 & pT2cNOM0 & $3+4=7$ & Adenocarcinoma & 8.9 \\
\hline 16 & 75 & pT1cN0M0 & $4+4=8$ & Adenocarcinoma & 5.1 \\
\hline 17 & 67 & pT2cNOM0 & $3+5=8$ & Adenocarcinoma & 29.1 \\
\hline 18 & 73 & pT3aN0M0 & $3+5=8$ & Adenocarcinoma & 7.4 \\
\hline 19 & 67 & pT2cN0M0 & $3+4=7$ & Adenocarcinoma & 7.2 \\
\hline 20 & 51 & pT2cN0M0 & $3+4=7$ & Adenocarcinoma & 4.8 \\
\hline 21 & 61 & pT3aN0M0 & $4+3=7$ & Adenocarcinoma & 8.2 \\
\hline
\end{tabular}

${ }^{a}$ pTNM was based on the 7th edition of the TNM classification of malignant tumours (Wiley-Blackwell, 2009). ${ }^{\mathrm{b}}$ Gleason score was assigned according to the 2014 ISUP consensus, appeared in Am J Surg Pathol 40(2): 244-52, 2016. PSA, prostate-specific antigen.

summary of the sequencing statistics as a representative case of the PCaFusion panel analysis is shown in Fig. S1B. The mean number of obtained reads was 539,860 . The data on the average alignment ratios revealed that $88.1 \%$ of the total reads were aligned properly to the hg19 human genome reference sequence.

Gene variants by KCC71 panel analysis. As indicated in Materials and methods, confirmed somatic nucleotide sequence alterations of non-synonymous SNVs and indels, with or without frameshifts, were considered as somatic variants in the present study. Somatic variants were detected in 17 of 21 patients by the present panel analyses. No variants were detected in 4 patients (cases 18-21). A total of 33 somatic variants were identified in 24 of $71 \mathrm{PCa}$-related genes in the KCC71 panel. The results are summarized in Fig. 1 (detailed information is provided in Table SIII).

A total of 7 probable pathogenic variants in 5 genes were identified in the present KCC71 panel analyses. Evident driver gene variants in the literature were found in $B R A F$ (p.K601E) (22) and SPOP (p.F102V and p.F133L) $(5,6)$. Although not well characterized as driver genes in the literature, somatic variants with a high pathogenic score predicted by FATMM (23-25) were also identified in $C D H 1$ (p.E880K) and $R B 1$ (p.R621S). Two variants found in BRCA2, namely p.I1859fs and p.R2318ter, which may result in premature termination and truncation of the BRCA 2 polypeptide, were considered as pathogenic, although the identical alterations did not appear in COSMIC.

The remaining 26 variants were considered as variants of uncertain/unknown significance (VUSs), including $A R$ (p.K610E), CDH1 (p.G62V), FOXAl (p.R265-K267 del) and TP53 (p.V31I). These variants were found in the COSMIC v82 database with labels of 'n/a' or 'neutral' based on FATHMM score. The CDH1 (p.G62V) variant is not registered in COSMIC; however, the identical mutation was reported as a germline mutation detected in families with hereditary diffuse gastric cancer (26). This non-synonymous mutation was in a region encoding a pro-domain and is generally considered to be non-pathogenic (23-25). The remaining 21 somatic mutations did not appear in COSMIC.

Fusion transcripts by the PCaFusion panel analysis. The existence of gene fusion transcripts was analyzed to identify the presence of fusion genes with the original PCaFusion panel. All 8 non-fusion transcripts evaluated as positive controls were detected in all specimens. In the present study, fusion transcripts were designated as follows: The 5' gene symbol (number of the exon located at the fusion site)-the $3^{\prime}$ partner gene symbol (exon number). For SLC45A3-ELK4 fusion transcripts, SLC45A3(1)-ELK4(2) and SLC45A3(1)-ELK4(4) were detected in all cases. By contrast, SLC45A3(2)-ELK4(2) was identified in only 1 case (case 5). USP9Y-TTTY15 fusion transcripts were detected in all examined cases. Among the 


\begin{tabular}{|c|c|c|c|c|c|c|c|c|c|c|c|c|c|c|c|c|c|c|c|c|c|c|c|}
\hline & & Genes & 1 & 2 & 3 & 4 & 5 & 6 & 7 & 8 & 9 & 10 & 11 & 12 & 13 & 14 & 15 & 16 & 17 & 18 & 19 & 20 & 21 \\
\hline \multirow{24}{*}{ 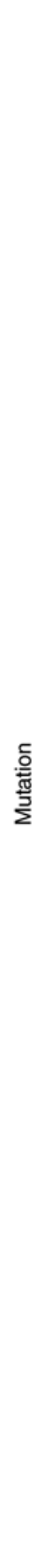 } & \multirow{2}{*}{$\frac{\mathrm{m}}{a}$} & AKT1 & & & & & & & & & & & & & & & & & & & & & \\
\hline & & PHLPP1 & & & & & & & & & & & & & & & & & & & & & \\
\hline & \multirow{2}{*}{$\underset{\nwarrow}{\infty}$} & BRAF & & & & & & & & & & & & & & & & & & & & & \\
\hline & & RAF1 & & & & & & & & & & & & & & & & & & & & & \\
\hline & \multirow{5}{*}{$\frac{⿱}{4}$} & AR & & & & & & & & & & & & & & & & & & & & & \\
\hline & & TNK2 & & & & & & & & & & & & & & & & & 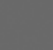 & & & & \\
\hline & & ELAC2 & & & & & & & & & & & & & & & & & & & & & \\
\hline & & FOXA1 & & & & & & & & & & & & & & & & 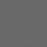 & & & & & \\
\hline & & KDM4B & & & & & & & & & & & & & & & & & & & & & \\
\hline & \multirow{3}{*}{ 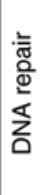 } & BRCA1 & & & & & & & & & & & & & & & & & & & & & \\
\hline & & BRCA2 & & & & & & & & & & & & & & & & & & & & & \\
\hline & & ATBF1 & & & & & & & & & & & & & & & & & & & & & \\
\hline & \multirow{4}{*}{$\begin{array}{l}\frac{\Phi}{0} \\
\text { তे } \\
\overline{\bar{\Phi}}\end{array}$} & TP53 & & & & & & & & & & & & & & & & & & & & & \\
\hline & & $\mathrm{CDH} 1$ & & & & & & & & & & & & & & & & & & & & & \\
\hline & & CDK2 & & & & & & & & & & & & & & & & & & & & & \\
\hline & & RB1 & & & & & & & & & & & & & & & & & & & & & \\
\hline & \multirow{8}{*}{ 迹 } & SPOP & & & & & & & & & & & & & & & & & & & & & \\
\hline & & C14orf49 & & & & & & & & & & & & & & & & & & & & & \\
\hline & & THSD7B & & & & & & & & & & & & & & & & & & & & & \\
\hline & & ZNF595 & & & & & & & & & & & & & & & & & & & & & \\
\hline & & LRP1B & & & & & & & & & & & & & & & & & & & & & \\
\hline & & FAT4 & & & & & & & & & & & & & & & & & & & & & \\
\hline & & HMCN1 & & & & & & & & & & & & & & & & & & & & & \\
\hline & & sox9 & & & & & & & & & & & & & & & & & & & & & \\
\hline \multirow{5}{*}{$\frac{}{\frac{0}{n}}$} & \multirow{5}{*}{ 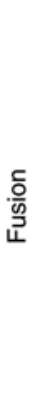 } & $\begin{array}{l}\text { USP9Y(3)- } \\
\text { TTTY15(3) }\end{array}$ & & & & & & & & & & & & & & & & & & & & & \\
\hline & & $\begin{array}{l}\text { SLC45A3(1)- } \\
\text { ELK4(2) }\end{array}$ & & & & & & & & & & & & & & & & & & & & & \\
\hline & & $\begin{array}{c}\text { SLC45A3(1)- } \\
\text { ELK4(4) }\end{array}$ & & & & & & & & & & & & & & & & & & & & & \\
\hline & & $\begin{array}{c}\text { SLC45A3(2)- } \\
\text { ELK4(2) }\end{array}$ & & & & & & & & & & & & & & & & & & & & & \\
\hline & & $\begin{array}{l}\text { TMPRSS2(1)- } \\
\text { ERG(4) }\end{array}$ & & & & & & & & & & & & & & & & & & & & & \\
\hline
\end{tabular}

Figure 1. Analyses of somatic variants and fusion transcripts by panel sequencing. The vertical axis indicates gene symbols or fusion transcripts. Genes in the same pathway are in the same column. The horizontal axis represents each patient's ID. Shaded boxes, pathogenic variants; gray boxes, non-synonymous variants of uncertain significance. 
Table II. Comparison of the frequency of somatic variants identified between the KCC71 analysis and TCGA database evaluated by signaling pathway.

\begin{tabular}{lccc}
\hline $\begin{array}{l}\text { Signaling } \\
\text { pathway }\end{array}$ & KCC $(\%)$ & TCGA $(\%)$ & $\begin{array}{c}\text { P-value } \\
\text { (Fisher's log10) }\end{array}$ \\
\hline PI3K & 9.5 & 9.4 & 0.22 \\
RAS & 9.5 & 3.6 & 0.72 \\
AR & 28.6 & 6.2 & 2.67 \\
DNA Repair & 28.6 & 2.6 & 4.40 \\
Cell cycle & 23.8 & 17.8 & 0.49 \\
other & 52.4 & 33.2 & 1.20 \\
\hline
\end{tabular}

PI3K, phosphoinositide 3 kinase; RAS, rat sarcoma oncogene; AR, androgen receptor; TCGA, The Cancer Genome Atlas.

TMPRSS2-ERG fusion gene transcripts, TMPRSS2(1)-ERG(4) was identified in only one case (case 11). No other fusion transcripts were identified in the PCaFusion panel analysis. The results are summarized in Fig. 1.

Comparative analysis between the variants detected by the panels and the variants registered in cBioPortal. A comparative analysis of the results of the KCC71 panel with TCGA and other big data registered in cBioPortal (http://www.cbioportal. org) was performed. Briefly, the frequency of somatic variants in the aforementioned public databases were examined, including the databases of TCGA, Broad Institute, Freed Hutchinson Cancer Research Center, and Memorial Sloan Kettering Cancer Center (hereafter referred to as 'cBioPortal databases') for the 71 genes in the KCC71 panel, and this was compared with the frequency obtained in the present study. The total frequency of somatic variants in the 71 genes, calculated as the total variant number identified per examined case, was higher compared with that in the cBioPortal databases (present study, 33 different variants in 21 cases; summary of cBioPortal databases, 849 variants in 1,656 cases) (Fig. S2, and Tables SIV and SV). This difference was particularly notable for the frequencies of variants in $A T B F 1, B R C A 2$ and $L R P 1 B$, which were all $\geq 10 \%$ compared with those in the cBioPortal databases. By contrast, the frequency of variants of TP53 was low (1 in 21 cases, $4.8 \%$; Fig. 1). To compare those mutation frequencies in terms of pathways, the ratio between the number of patients with and without mutations in the genes in a particular pathway was calculated. Then, the ratios from our database and TCGA databases were compared using Fisher's exact test. We observed that those ratios in genes belonging to the androgen receptor (AR; - $\log 10$ Fisher's P-value=2.67) and DNA Repair (-log10 Fisher's P-value=4.40) signaling pathways were particularly high compared with those in TCGA database (Tables II, SIV and SV).

Pathways of PCa predicted by TCNG network analysis. Our network analysis consisted of four steps as explained below (Fig. 2A-D). In the first step, genes with mutations from the KCC71 panel analysis were selected as 'initial seed genes' (Fig. 2A). In the second step, the 7 gene networks of $\mathrm{PCa}$ in TCNG were selected as graphical representations of the regulatory relationships between genes. The Gene Expression Omnibus (GEO) ID and information from each selected gene network for PCa are shown in Fig. 2B and Table SVI. In the third step, the 'initial seed genes' were mapped on the 7 PCa gene networks, and a subnetwork around the 'initial seed genes' was extracted from each of the gene networks; each subnetwork consisted of the 'initial seed genes' and downstream genes within the two passes around the initial seed genes (Fig. 2C). Genes with one path from 'initial seed genes' are referred to as 'child genes' and genes with a path from the child genes are referred to as 'grandchild genes'. The obtained subnetworks show the regulation around the seed genes. Next, we attempted to identify 'extended common seed genes' that are shared among $\geq 6$ subnetworks. In the last step, a putative 'core network' of PCa was estimated by integrating subnetworks around 'the extended common seed genes,' referred to as 'extended subnetworks' (Fig. 2D). The above network operations were conducted by using the functions of igraph, a package of $\mathrm{R}$ version 3.5.0. The subnetworks (relationships) of gene regulation were demonstrated by graphical visualization using Cytoscape software version 3.5.1 (19), as shown in Fig. S3, and the core network was presented using igraph.

Two publicly available tools, Database for Annotation, Visualization, and Integrated Discovery (DAVID; Laboratory of Human Retrovirology and Immunoinformatics, https:// david.ncifcrf.gov/home.jsp) (20) and Reduce + Visualize Gene Ontology (REVIGO; Redjer Boskivic Institute; http:// revigo.irb.hr) (21), were then used to investigate the biological functions associated with the gene groups involved in the core network. DAVID v6.8, which mainly provides typical batch annotation and Gene Ontology (GO) term enrichment analysis, was used to highlight the most relevant GO terms associated with a given gene list, in order to elucidate the biological meaning behind a large list of genes. Enrichment analysis was performed using DAVID for the core network genes listed in Table SVII, and a functional annotation chart including a list of GO IDs and P-values for the enrichment tests was obtained. The functional annotation chart report of DAVID shows categories, enriched terms associated with a gene list of interest, related term search, genes involved in the term, and percentages or modified Fisher's exact P-values. REVIGO was used to summarize the results obtained from DAVID (21). REVIGO provides a functional interpretation of genes defined by $\mathrm{GO}$ with statistical methods. A list of GO IDs and P-values was entered from the functional annotation chart report of DAVID. The REVIGO GO tree map shows a two-level hierarchy of GO terms.

The workflow for the reconstruction of the core network of PCa was schematically summarized with TCNG (Fig. 2A-D). The 24 genes with somatic variations from the KCC71 panel analysis (Fig. 1) were selected as 'initial seed genes.' Although only 5 well-characterized pathogenic driver gene mutations were identified in the present analysis and the remaining 19 genes were considered as VUSs, all 'initial seed genes' were reported to be involved in $\mathrm{PCa}$ in the literature and databases (Materials and methods, Sample preparation and target sequencing with the original panels). Subnetworks were extracted from 7 public PCa gene networks in TCNG 
A

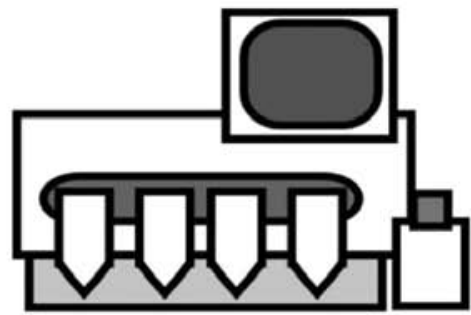

24 genes with mutations

from the KCC71 panel selected as "initial seed genes"
B

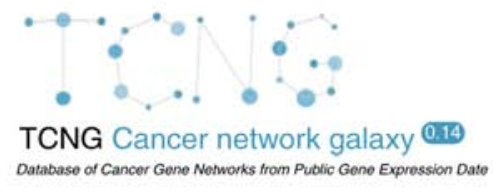

- GSE6956 • GSE8218

- GSE14206 • GSE16120

- GSE17951 • GSE25136

- GSE32269

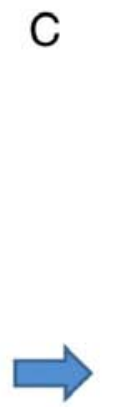

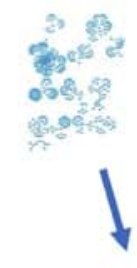

Extended common seed genes

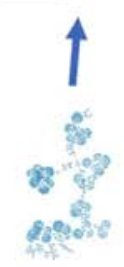

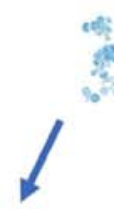

$\sim$

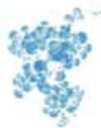

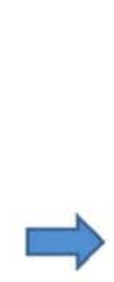

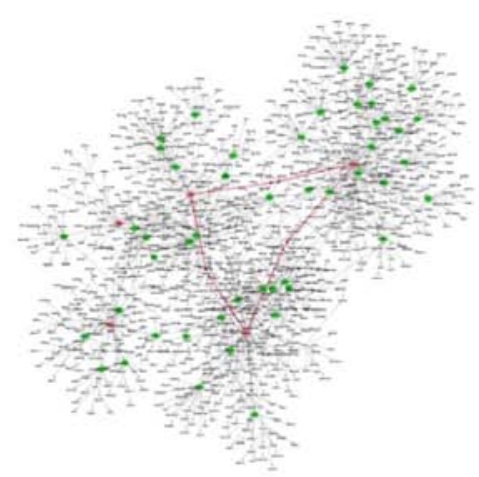

Figure 2. Network analysis of prostate cancer tumorigenesis scheme to reconstruct a core network of prostate cancer with TCNG database. (A) Detection of initial seed genes through somatic variant analysis. (B) Selection of prostate cancer-related networks from TCNG, followed by the extraction of subnetworks centered on each initial seed gene. (C) Extraction of extended common seed genes from multiple subnetworks. (D) Construction of a core network from the extended common seed genes. TCNG, The Cancer Network Galaxy.

(Table SVI), which included initial seed genes (parent nodes), and parent-child and child-grandchild genes in the network (Fig. S3). 'Extended common seed genes', TNK2, SOX9, CDH1, FOXA1 and TP53, that were commonly included in the subnetworks, were extracted. To identify the core network of PCa s examined, extended subnetworks around the 'extended common seed genes' were further extracted from each of the original 7 PCa networks, integrated, and the core network was finally reconstructed.

The core network around the 'extended common seed genes' was further analyzed. The 3 extended common seed genes, $S O X 9, C D H 1$ and $F O X A 1$, were connected via edges with each other through the genes $E M X 2, N K X 3-1$ and TFAP2A, and formed a closed loop (Fig. 3, red arrows). $E M X, N K X 3-1$ and TFAP $2 A$ were the only genes in the network located between the extended common genes. The top 15 genes with high connectivity (hub genes), are listed in Table SVIII. All 5 extended common genes are listed in Table SIV, but none of the initial seed genes appears in it. Only $A R$ and $S P O P$ as the initial seed genes appear in the final network, with few edges.

The enrichment analysis using DAVID and REVIGO found 50 GO terms with adjusted P-values (Table SIX). REVIGO generates tree maps of the GO terms, as shown in Fig. 4 and Fig. S4. The GO terms are joined into 'superclusters' of loosely related terms and depicted with different colors. The most significant GO term found was 'positive regulation of transcription from RNA polymerase II promoter', followed by 'epithelial cell differentiation', 'response to water deprivation', 'tissue homeostasis', and 'amino acid transport'.

\section{Discussion}

The present study attempted to elucidate the molecular pathways involved in PCa in Japanese patients by starting with a limited number of cases and with a new bioinformatics analysis using TCNG. Starting the analysis with 24 genes harboring mutations as initial seed genes, we reached a core network involving 3 genes that were not included among the initial seed genes, but 2 of those had been well-characterized in relation to $\mathrm{PCa}$. This may demonstrate the validity of this analytical approach, but further estimation with a larger numbers of cases is required.

In the present study, 21 surgically removed PCa specimens without any neoadjuvant treatments were analyzed using our original DNA and RNA panels for PCa profiling. Both panels functioned appropriately, as revealed by sequencing statistics and the results obtained with the positive control set for the RNA panel. The well-characterized pathogenic TMPRSS2-ERG fusion gene transcript was identified in only 


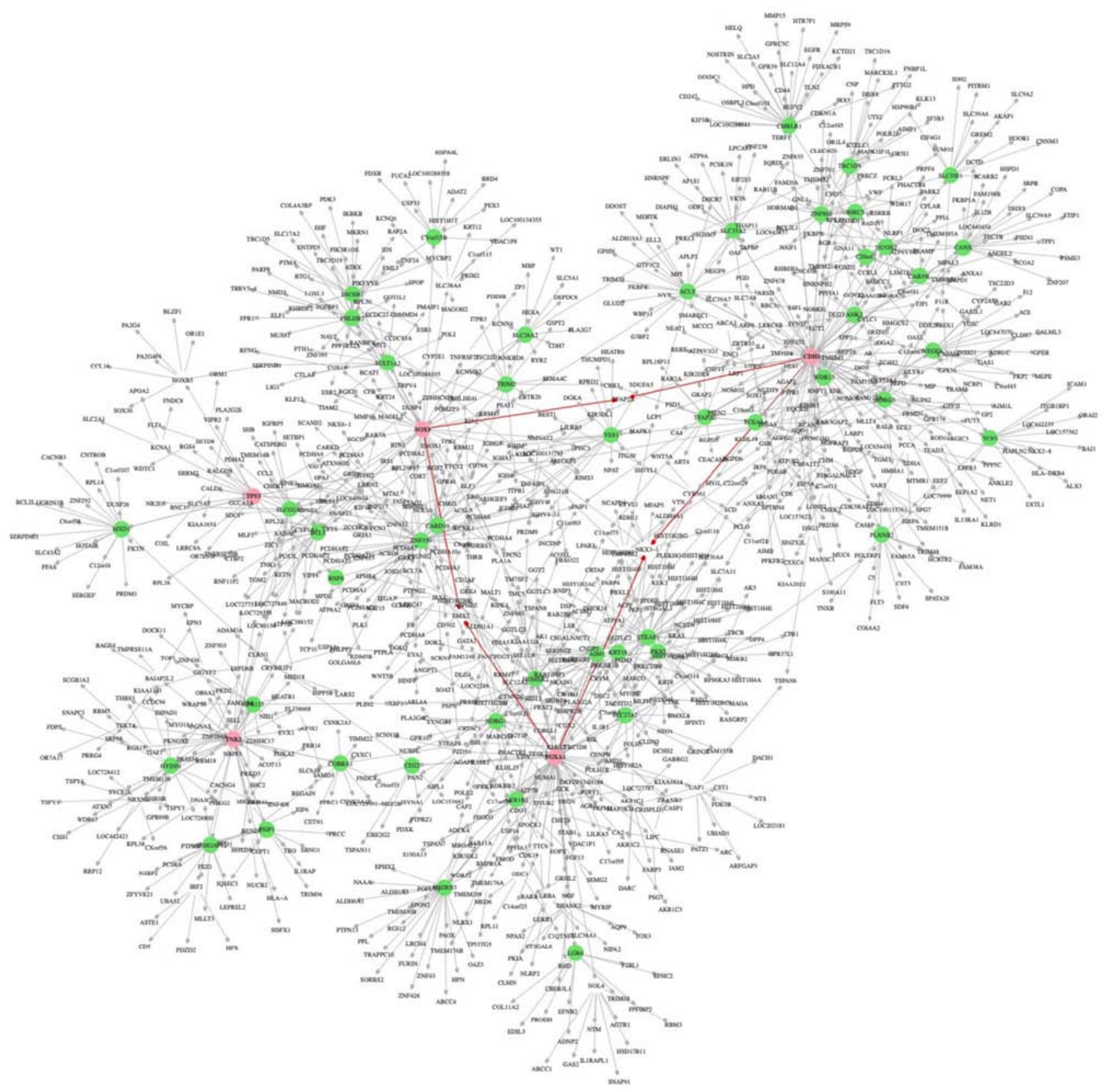

Figure 3. Predicted final core network for prostate cancer in the Japanese population. The network from seven shared prostate cancer networks is shown. Genes are represented by nodes with gene symbols and regulatory associations between genes are represented by arrows referred to as 'edges.' Edges that form a closed loop connecting the extended seed genes are colored red. Pink nodes, 5 extended seed genes; green nodes, nodes with $\geq 10$ edges.

1 case $(1 / 21,4.8 \%)$ in the PCaFusion panel analysis, which was an unexpectedly low frequency when compared with that in previous reports, even in Japanese or Chinese cohorts in which the rate was significantly lower compared with that in cohorts from Western countries $(9,27)$. By contrast, two other transcription-mediated chimeric RNAs, SLC45A3-ELK4 and USP9Y-TTTY15 fusion transcripts, were detected in all examined cases. Although enriched in cancer tissues, the pathogenicity of these fusion RNAs remains unclear, and they were found to be expressed in both cancerous and adjacent non-cancerous prostatic tissues. Highly sensitive methods, such as RT-PCR, have demonstrated these RNAs in almost all examined specimens $(28,29)$. As our PCaFusion panel analysis is a PCR-mediated amplicon sequencing technology, the obtained results were compatible with those in previous reports. A similar transcription-mediated chimeric RNA, SDK1-AMACR, was not found in the present study, although Chinese cohorts identified the fusion transcript in $23-24 \%$ of examined cases $(26,29)$. Despite their similar origins in East Asia, Chinese and Japanese PCa patients appear to differ in their genetic or epigenetic background.

The KCC71 panel analysis identified 33 different genetic variants associated with $\mathrm{PCa}$ in the Japanese. Two cases contained SPOP mutations $(2 / 21,9.5 \%)$ in the hotspots in the MATH domain. SPOP, encoding the E3 ubiquitin ligase, is the most frequently mutated gene, with mutations found in $6-15 \%$ of PCa cases across multiple cohorts, in a manner mutually exclusive with the presence of the fusion gene TMPRSS2-ERG. SPOP mutation is known to be associated with certain clinicopathological characteristics, such as 


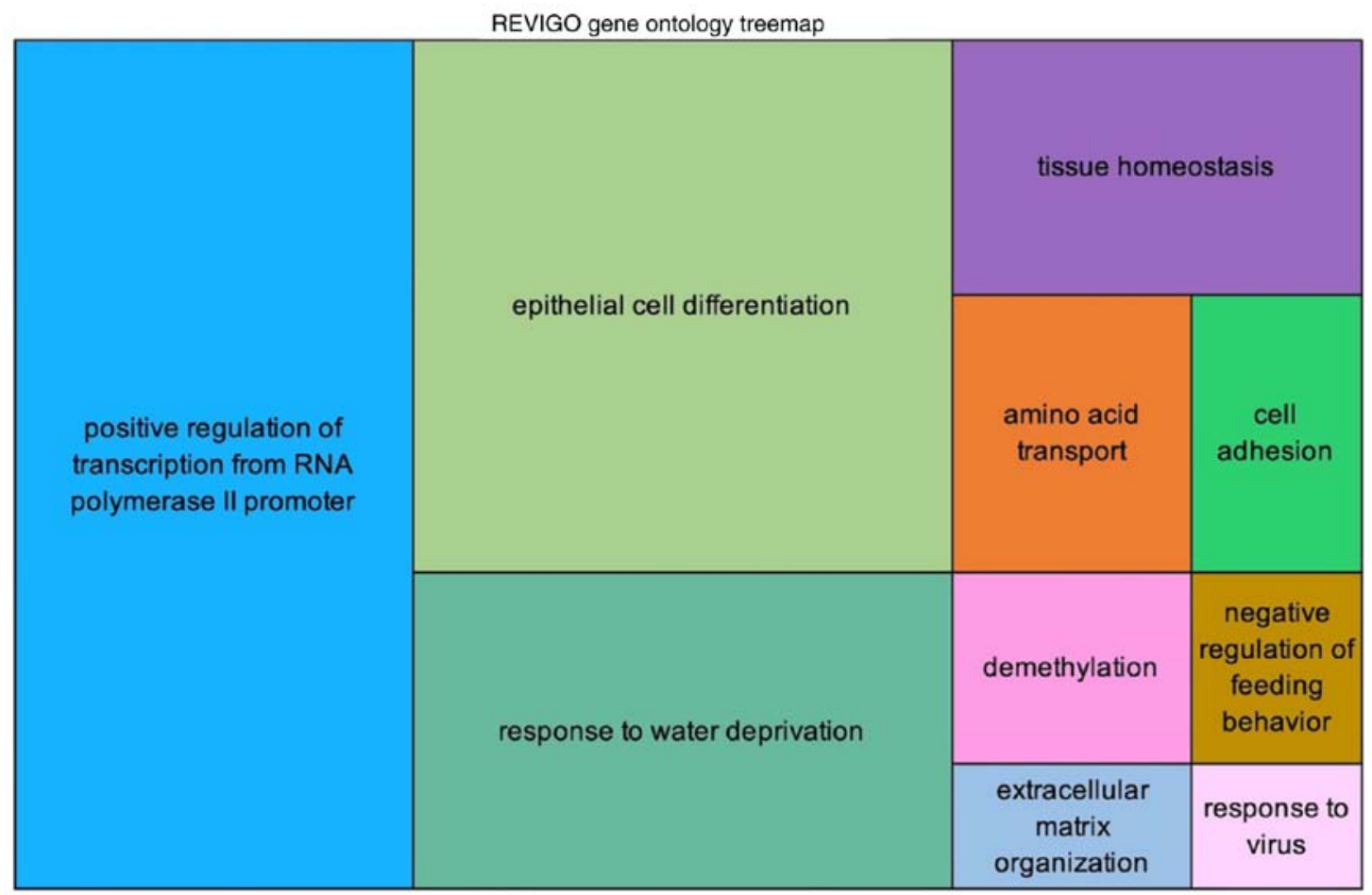

Figure 4. Biological processes of the core network of prostate cancer. REVIGO tree map showing the predicted biological processes of prostate cancer in the Japanese. Each rectangle represents a biological function in terms of a Gene Ontology (GO) term, with the size adjusted to represent the P-value of the GO term in the underlying GO term database. Superclusters are differentially colored. More detailed information is available in Fig. S4 and Table SVIII. REVIGO, Reduce + Visualize Gene Ontology.

serum PSA level, pathological parameters and patient prognosis $(6,11)$. The frequency of $S P O P$ mutation in the present study was comparable with that in previous reports, which may indicate the absence of major bias in our cohort; however, the reason for the low frequency of TMPRSS2-ERG is unclear. $B R C A 2$ truncating inactivating mutations were also identified in 2 cases $(9.5 \%)$. BRCA2 mutation is rare, with a frequency of $\sim 2 \%$ in early-onset $\mathrm{PCa}(30)$; it has also been shown to be associated with a higher Gleason score and poor prognosis $(31,32)$. Regarding our $B R C A 2$-mutated cases, one had a Gleason score of 9 and the other had a score of 7. The evaluation of $B R C A 2$ mutation with biopsy or surgical specimens may also be useful for selecting the treatment modality for Japanese patients.

Other pathogenic variants were found in $C D H 1, B R A F$ and $R B 1$, with 1 mutation per gene. The sample size was small and precise comparison of the mutation frequency of each gene with that in previous cohorts was not the principal objective of this research. However, the overall frequency of mutated genes and somatic variations in the 71 selected genes was higher compared with that calculated from public big data, such as TCGA. This may be a characteristic of PCa in Japanese patients, but further investigation in large cohorts is required.

In recent years, efforts have intensified to obtain novel meaningful insights into biological pathways involved in cancer by utilizing big data in the life sciences. We herein attempted to develop a new approach to extracting a common core network related to $\mathrm{PCa}$ in the Japanese population by integrating information on mutated genes identified in KCC71 panel analysis and multiple gene networks of PCa in TCNG. Subsequently, we developed a new way of exploring cancer-related gene interactions. TCNG is a database of cancer gene regulatory networks estimated from publicly available cancer gene expression data, in the GEO database, by using Bayesian network models $(12,13)$. In addition, by combining data and examining common genes that constitute the core network, it is possible to characterize the interactions among genes that may cause cancer. The core network may be considered as the center of the pathogenic pathway.

The central genes of the network estimated here were identified as the 'extended common seed genes' of PCa. All 5 identified common genes were initial seed genes and have been well characterized as being associated with cancer, including PCa. Surprisingly, only 3 genes were revealed to connect common genes to each other, namely TFAP2A (between $C D H 1$ and SOX9), EMX2 (between SOX9 and FOXA1), and NKX3-1 (between FOXAl and $C D H I$ ). Although none of these 3 genes was involved with the initial seed genes, $N K X 3-l$ is a well-known prostate-specific tumor suppressor (33) and has been implicated in prostatic epithelial cell differentiation (34) and the maintenance of luminal stem cells (35). TFAP2A, also referred to as $A P-2$ or $A P 2 T F / T F A P 2$, is a transcription factor and its tumor suppressor properties were also reported in cancers including PCa (36-38). As neither NKX3-1 nor TFAP2A were involved with the initial seed genes, which were the starting point of the present analysis, this may support the reliability of this analysis. By contrast, although $E M X 2$, a homeobox-containing transcription factor, was characterized as a tumor suppressor gene in cancers such as colorectal cancer (39), malignant pleural mesothelioma or lung cancer $(40,41)$, to the best of our knowledge no report on PCa has yet been published. Research on EMX2 may elucidate the biological/pathological characteristics of $\mathrm{PCa}$ in Japanese patients. 
In comparison with the generally Caucasian cohorts in TCGA, the involvement of the AR pathway and the DNA repair pathway were identified as characteristics associated with $\mathrm{PCa}$ in the Japanese population. Although the AR pathway is clearly significant worldwide, the potential involvement of the DNA repair pathway in this disease was identified due to the two pathogenic mutations that were identified in BRCA2. Momozawa et al (43) reported the results of germline mutation analysis of 7,636 Japanese PCa patients, and found that the BRCA2, but not $B R C A 1$, germline pathogenic variant was significantly associated with PCa in Japanese patients. This contradicts the Philadelphia Prostate Cancer Consensus 2017 (42), based generally on data from Western countries, which asserted that there is high-grade evidence on the association of both $B R C A l$ and $B R C A 2$ with $\mathrm{PCa}$ (43). It is possible that the disturbance of DNA repair, partly through the inactivation of $B R C A 2$, but not $B R C A 1$, is involved in PCa in Japanese patients. BRCA1 and BRCA2 are currently considered as homologous recombination-related genes, and associated differences in pathological phenotypes or the clinical significance of their mutations, such as sensitivity to poly(ADP-ribose) polymerase inhibitors, have not yet been well addressed (44). BRCA2 may warrant further research as a gene potentially associated with $\mathrm{PCa}$ in the Japanese.

We herein analyzed the associations among limited numbers of genes with somatic variations based on a Bayesian network model. Using a statistical approach, it appeared possible to predict the association among not only directly interacting genes, but also ones that act indirectly. The analysis using a statistical model may be effective when, for example, used to predict drug targets, as it can predict signaling pathways even from genes that are not directly associated with each other. In the analyses, genes that do not harbor well-characterized pathogenic mutations served as initial seed genes. Mutations with uncertain significance in these genes should be further functionally characterized in future research. In addition, although PCas are known to be clonally heterogeneous tumors, the heterogeneity was not considered in the present study. Additional larger studies considering this heterogeneity are required to obtain an overall understanding of PCa in the Japanese population.

\section{Acknowledgements}

The authors would like to thank the members of the Kanagawa Cancer Center Research Institute, Health Intelligence Center, Human Genomic Center, the Institute of Medical Science, University of Tokyo. Supercomputing resources were provided by Human Genome Center, University of Tokyo.

\section{Funding}

The present study was supported by Grants-in-Aid for Scientific Research (KAKENHI, nos. 17K1168, 16K19099 and 26860253).

\section{Availability of data and materials}

All data generated or analyzed during the present study are included in this published article. Sequence data are available upon request to the corresponding author; the request must include a description of the research proposal.

\section{Authors' contributions}

RK, YM, RY designed the study and wrote the manuscript; RK performed research and analyzed the data with the assistance of ES; TK, YM, IA and HU prepared the clinical samples and analyzed patient information; YT, SI, RY, AN, GT and ES prepared the data from TCNG database and supervised the analyses. SI, RY and SM supervised the research.

\section{Ethics approval and consent to participate}

The ethical committees for investigations with human materials at Yokohama City University Graduate School of Medicine and Kanagawa Cancer Center approved the study. Informed consent was obtained from all the participants.

\section{Patient consent for publication}

Not applicable.

\section{Competing interests}

The authors declare that they have no competing interests.

\section{References}

1. Tomlins SA, Rhodes DR, Perner S, Dhanasekaran SM, Mehra R, Sun XW, Varambally S, Cao X, Tchinda J, Kuefer R, et al: Recurrent fusion of TMPRSS2 and ETS transcription factor genes in prostate cancer. Science 310: 644-648, 2005.

2. Perner S, Demichelis F, Beroukhim R, Schmidt FH, Mosquera JM, Setlur S, Tchinda J, Tomlins SA, Hofer MD, Pienta KG, et al: TMPRSS2:ERG fusion-associated deletions provide insight into the heterogeneity of prostate cancer. Cancer Res 66: 8337-8341, 2006.

3. Helgeson BE, Tomlins SA, Shah N, Laxman B, Cao Q, Prensner JR, Cao X, Singla N, Montie JE, Varambally S, et al: Characterization of TMPRSS2:ETV5 and SLC45A3:ETV5 gene fusions in prostate cancer. Cancer Res 68: 73-80, 2008.

4. Kumar-Sinha C, Tomlins SA and Chinnaiyan AM: Recurrent gene fusions in prostate cancer. Nat Rev Cancer 8: 497-511, 2008.

5. Attard G,Parker C, Eeles RA, Schröder F, Tomlins SA, Tannock I, DrakeZZCG and de Bono JS: Prostate cancer. Lancet 387: 70-72, 2016.

6. Barbieri CE, Baca SC, Lawrence MS, Demichelis F, Blattner M, Theurillat JP, White TA, Stojanov P, Van Allen E, Stransky N, et al: Exome sequencing identifies recurrent SPOP, FOXA1 and MED12 mutations in prostate cancer. Nature Genet 44: 685-689, 2012.

7. Cavanagh $\mathrm{H}$ and Rogers KM: The role of $B R C A 1$ and $B R C A 2$ mutations in prostate, pancreatic and stomach cancers: Hered Cancer Clin Pract 1: 13: 16, 2015.

8. Witte JS, Mefford J, Plummer SJ, Liu J, Cheng I, Klein EA, Rybicki BA and Casey G: HOXB13 mutation and prostate cancer: Studies of siblings and aggressive disease. Cancer Epidemiol Biomarkers Prev 22: 675-680, 2013.

9. Miyagi Y, Sasaki T, Fujinami K, Sano J, Senga Y, Miura T, Kameda Y, Sakuma Y, Nakamura Y, Harada M and Tsuchiya E: ETS family-associated gene fusions in Japanese prostate cancer: analysis of 194 radical prostatectomy samples. Mod Pathol 23: 1492-1498, 2010.

10. Haffner MC, Mosbruger T, Esopi DM, Fedor H, Heaphy CM, Walker DA, Adejola N, Gürel M, Hicks J, Meeker AK, et al: Tracking the clonal origin of lethal prostate cancer. J Clin Invest 123: 4918-4922, 2013.

11. Shoag J, Liu D, Blattner M, Sboner A, Park K, Deonarine L, Robinson BD, Mosquera JM, Chen Y, Rubin MA and Barbieri CE: SPOP mutation drives prostate neoplasia without stabilizing oncogenic transcription factor ERG. J Clin Invest 128: 381-386, 2018

12. Tamada Y, Imoto S, Araki H, Nagasaki M, Print C, Charnock-Jones DS and Miyano S: Estimating genome-wide gene networks using nonparametric Bayesian network models on massively parallel computers. IEEE/ACM Trans Comput Biol Bioinform 8: 683-697, 2011. 
13. Imoto $\mathrm{S}$, Goto $\mathrm{T}$ and Miyano $\mathrm{S}$ : Estimation of genetic networks and functional structures between genes by using Bayesian network and nonparametric regression. Pac Symp Biocomput 7: 175-186, 2002.

14. Huang J, Wang JK and Sun Y: Molecular pathology of prostate cancer revealed by next-generation sequencing: Opportunities for genome-based personalized therapy. Curr Opin Urol 23 189-193, 2013.

15. Beltran H, Yelensky R, Frampton GM, Park K, Downing SR, MacDonald TY, Jarosz M, Lipson D, Tagawa ST, Nanus DM, et al: Targeted next-generation sequencing of advanced prostate cancer identifies potential therapeutic targets and disease heterogeneity. Eur Urol 63: 920-926, 2013.

16. Zhu Y, Ren S, Jing T, Cai X, Liu Y, Wang F, Zhang W, Shi X, Chen R, Shen J, et al: Clinical utility of a novel urine-based gene fusion TTTY15-USP9Y in predicting prostate biopsy outcome. Urol Oncol 33: 384.e9-20, 2015.

17. St John J, Powell K, Conley-Lacomb MK and Chinni SR: TMPRSS2-ERG fusion gene expression in prostate tumor cells and its clinical and biological significance in prostate cancer progression. J Cancer Sci Ther 4: 94-101, 2012.

18. Rubin MA, Maher CA and Chinnaiyan AM: Common gene rearrangements in prostate cancer. J Clin Oncol 29: 3659-3668, 2011.

19. Shannon P, Markiel A, Ozier O, Baliga NS, Wang JT, Ramage D, Amin N, Schwikowski B and Ideker T: Cytoscape: A software environment for integrated models of biomolecular interaction networks. Gnome Res 13: 2498-2504, 2003.

20. Jiao X, Sherman BT, Huang da W, Stephens R, Baseler MW, Lane HC and Lempicki RA: DAVID-WS: A stateful web service to facilitate gene/protein list analysis. Bioinformatics 28 : 1805-1806, 2012.

21. Supek F, Bošnjak M, Škunca N and Šmuc T: REVIGO summarizes and visualizes long lists of Gene Ontology terms. PLoS One 6: e21800, 2011.

22. Menzies AM and Long GV: Long, dabrafenib and trametinib, alone and in combination for BRAF-mutant metastatic melanoma. Clin Cancer Res 20: 2035-2043, 2014.

23. Shihab HA, Gough J, Cooper DN, Stenson PD, Barker GL, Edwards KJ, Day IN and Gaunt TR: Predicting the functional, molecular and phenotypic consequences of amino acid substitutions using hidden Markov models. Hum Mutat 34: 57-65, 2013

24. Shihab HA, Gough J, Cooper DN, Day INM and Gaunt TR: Predicting the functional consequences of cancer-associated amino acid substitutions. Bioinformatics 29: 1504-1510, 2013.

25. Shihab HA, Gough J, Mort M, Cooper DN, Day IN and Gaunt TR: Ranking non-synonymous single nucleotide polymorphisms based on disease concepts. Hum Genom 8: 11, 2014.

26. Simões-Correia J, Figueiredo J, Lopes R, Stricher F, Oliveira C, Serrano L and Seruca R: E-Cadherin destabilization accounts for the pathogenicity of missense mutations in hereditary diffuse gastric cancer. PLoS One 7: e33783, 2012.

27. Ren S, Peng Z, Mao JH, Yu Y, Y in C, Gao X, Cui Z, Zhang J, Yi K, $\mathrm{Xu} \mathrm{W}$, et al: RNA-seq analysis of prostate cancer in the Chinese population identifies recurrent gene fusions, cancer-associated long noncoding RNAs and aberrant alternative splicings. Cell Res 22: 806-821, 2012.

28. Kumar-Sinha C, Kalyana-Sundaram S and Chinnaiyan AM: SLC45A3-ELK4 chimera in prostate cancer: Spotlight on cis-splicing. Cancer Discov 2: 582-585, 2012.

29. Zhang Y, Mao XY, Liu X, Song RR, Berney D, Lu YJ and Ren G: High frequency of the SDK1:AMACR fusion transcript in Chinese prostate cancer. Int J Clin Exp Med 8: 15127-15136, 2015.
30. Edwards SM, Kote-Jarai Z, Meitz J, Hamoudi R, Hope Q, Osin P, Jackson R, Southgate C, Singh R, Falconer A, et al: Two percent of men with early-onset prostate cancer harbor germline mutations in the BRCA2 gene. Am J Hum Genet 72: 1-12, 2003.

31. Mitra A, Fisher C, Foster CS, Jameson C, Barbachanno Y, Bartlett J, Bancroft E, Doherty R, Kote-Jarai Z, Peock S, Easton D, IMPACT and EMBRACE, et al: Prostate cancer in male BRCA1 and BRCA2 mutation carriers has a more aggressive phenotype. Br J Cancer 98: 502-507, 2008.

32. Cui M, Gao XS, Gu X, Guo W, Li X, Ma M, Qin S, Qi X, Xie M, Peng $\mathrm{C}$ and Bai Y: BRCA2 mutations should be screened early and routinely as markers of poor prognosis: Evidence from 8,988 patients with prostate cancer. Oncotarget 8: 40222-40232, 2017.

33. Abate-Shen C, Shen MM and Gelmann E: Integrating differentiation and cancer: The $N k x 3.1$ homeobox gene in prostate organogenesis and carcinogenesis. Differentiation 76: 717-727, 2008.

34. Dutta A, Magnen C, Mitrofanova A, Ouyang X, Califano A and Abate-Shen C: Identification of an NKX3.1-G9a-UTY transcriptional regulatory network that controls prostate differentiation. Science 352: 1576-1580, 2016.

35. Talos F, Mitrofanova A, Bergren SK, Califano A and Shen MM: A computational systems approach identifies synergistic specification genes that facilitate lineage conversion to prostate tissue. Nat Commun 8: 14662, 2017.

36. Ruiz M, Troncoso P Bruns C and Bar-Eli M: Activator protein 2alpha transcription factor expression is associated with luminal differentiation and is lost in prostate cancer. Clin Cancer Res 7: 4086-4095, 2001.

37. Ruiz M, Pettaway C, Song R, Stoeltzing O, Ellis L and Bar-Eli M: Activator protein 2alpha inhibits tumorigenicity and represses vascular endothelial growth factor transcription in prostate cancer cells. Cancer Res 64: 631-638, 2004.

38. Jonckheere N, Fauquette V, Stechly L, Saint-Laurent N, Aubert S, Susini C, Huet G, Porchet N, Van Seuningen I and Pigny P: Tumour growth and resistance to gemcitabine of pancreatic cancer cells are decreased by AP-2alpha overexpression. $\mathrm{Br}$ J Cancer 101: 637-644, 2009.

39. Aykut B, Ochs M, Radhakrishnan P, Brill A, Höcker H, Schwarz S, Weissinger D, Kehm R, Kulu Y, Ulrich A and Schneider M: $E M X 2$ gene expression predicts liver metastasis and survival in colorectal cancer. BMC Cancer 17: 555, 2017.

40. Giroux Leprieur E, Hirata T, Mo M, Chen Z, Okamoto J, Clement G, Li H, Wislez M, Jablons DM and He B: The homeobox gene $E M X 2$ is a prognostic and predictive marker in malignant pleural mesothelioma. Lung Cancer 85: 465-471, 2014.

41. Okamoto J, Hirata T, Chen Z, Zhou HM, Mikami I, Li H, Yagui-Beltran A, Johansson M, Coussens LM, Clement G, et al: EMX2 is epigenetically silenced and suppresses growth in human lung cancer. Oncogene 29: 5969-5975, 2010.

42. Giri VN, Knudsen KE, Kelly WK, Abida W, Andriole GL, Bangma $\mathrm{CH}$, Bekelman JE, Benson MC, Blanco A, Burnett A, et al: Roel of genetic testing for inherited prostate cancer risk: Philadelphia prostate cancer consensus conference2017. J Clin Oncol 36: 414-424, 2018

43. Momozawa Y, Iwasaki Y, Hirata M, Liu X, Kamatani Y, Takahashi A, Sugano K, Yoshida T, Murakami Y, Matuda K, et al: Germline pathogenic variants in 7636 japanese patients with prostate cancer and 12366 controls. J Natl Cancer Inst: Jun 19, 2019 (Epub ahead of print).

44. Venkitaraman AR: Cancer susceptibility and the functions of BRCA1 and BRCA2. Cell 108: 171-182, 2002. 